\title{
Software
}

\section{Publicar con estándares web mediante herramientas de coste cero: revisión de NVU, Pages y Blogger}

\author{
Por Lluís Codina
}

\begin{abstract}
Resumen: Existe una nueva preocupación de respeto por los estándares para publicar en la web. Al mismo tiempo se hallan disponibles algunas aplicaciones de coste cero (open source o freeware) que permiten publicar en internet a personas u organismos que de otro modo tal vez no podrían hacerlo. Se estudian en este trabajo tres de ellas: $N V U$, Pages y Blogger, que muestran diversos grados de preocupación por los estándares así como también diversos niveles de facilidad de uso. La conclusión es que se encuentran recursos técnicos y oportunidades suficientes para que nadie deba limitar su presencia en la web por otro motivo que no sea la calidad y la utilidad teórica de los contenidos.
\end{abstract}

Palabras clave: Estándares web, Buenas prácticas, Html, Xhtml, Css, NVU, Pages, Blogger.

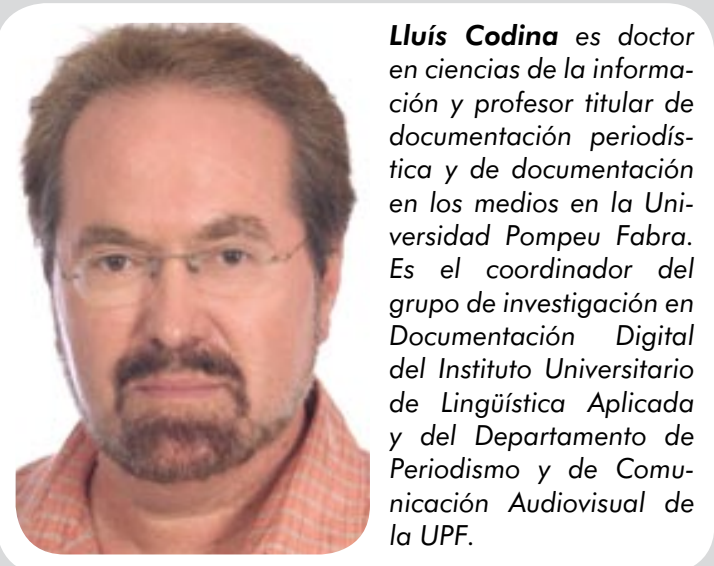

Lluís Codina es doctor la informadocumentación periodística y de documentación en los medios en la UniEompeu Fabra. el coordinador del en de investigación en Digital Aplicada nicación Audiovisual de la UPF.

\section{Title: Publishing with free web standards: a review of NVU, Pages and Blogger}

Abstract: Web publishing is experiencing a new emphasis on publication standards. At the same time, some no-cost applications (open source or freeware) allow web publishing by people and organisations that otherwise could not afford to do so. Three of these applications are discussed: NVU, Pages and Blogger. These three applications have various degrees of compliance with publishing standards and vary in terms of ease of use. The conclusion is that sufficient technical resources and publication opportunities exist so that any person or group's web presence is limited only by the quality of the concept and usefulness of the content.

Keywords: Web standards, Best practices, Html, Xhtml, Css, NVU, Pages, Blogger.

Codina, Lluís. "Publicar con estándares web mediante herramientas de coste cero: revisión de NVU, Pages y Blogger". En: El profesional de la información, 2007, julio-agosto, v. 16, n. 4, pp. 368-374.

DOI: $10.3145 /$ epi.2007.jul.12

\section{Introducción}

\subsection{Oportunidad}

INCLUSO LAS BIBLIOTECAS O CENTROS DE DOCUMENTACIÓN de organismos medianos o grandes, con fama de recursos abundantes, pueden dar la bienvenida a formas de publicar y difundir información a coste cero: ni a nadie le amarga un dulce, ni ninguna organización inteligente obviará formas alternativas de difusión de sus contenidos.

Además, existen diferentes casuísticas de personas que se en- cuentran en la siguiente situación: publicar en la web les proporcionaría ventajas competitivas, pero no pueden permitirse el lujo ni de externalizar el encargo ni de hacer grandes inversiones en software ${ }^{1}$. ¿Deben éstos renunciar a tener su propia plataforma en la web? Estamos pensando, por ejemplo, en profesionales free-lance, en pequeñas empresas, o en estudiantes de tercer ciclo y jóvenes investigadores que podrían dar a conocer sus productos o resultados.

\subsection{Requerimientos}

El relativamente nuevo proyecto de una web semántica, precedi- do y seguido respectivamente por iniciativas como Dublin Core y los microformatos, así como la defensa de buenas prácticas en edición de páginas web, presionan desde hace

$$
\begin{aligned}
& \text { Sitios web de los } \\
& \text { productos analizados } \\
& \text { - NVU } \\
& \text { http://www.nvu.com } \\
& \text { - Pages } \\
& \text { http://pages.google.com } \\
& \text { - Blogger } \\
& \text { http://www.blogger.com }
\end{aligned}
$$


unos años a favor de sitios y de páginas desarrollados mediante lo que se ha dado en llamar marcado o código semántico.

La siguientes cosas como mínimo son consideradas como marcado semántico:

- Lenguaje estándar de codificación. Es decir, uso de las últimas recomendaciones del World Wide Web Consortium para estructurar páginas. Actualmente, esto significa usar Xhtml 1 o html 4. Ya que Xhtml en realidad es la versión de html 4 compatible con xml, a partir de ahora para referirnos indistintamente a estos dos lenguajes, usaremos html.

- No usar elementos y atributos depreciados (deprecated) de html. Si se quiere, esta condición en realidad es el reverso de la anterior; o sea, una lleva a la otra.

- Html para el contenido y css para la presentación. Las etiquetas del lenguaje html son para estructurar el contenido (título, párrafo, tabla, etc.) y css para especificar la tipografía, color, tamaño, posición, etc., de cada elemento de la página, es decir, para decidir su presentación.

- Uso adecuado de los elementos con valor semántico. Esto incluye aspectos tales como identificar la estructura y los componentes de la página con el uso adecuado de elementos html tan conocidos como $h 1, p$, etc., pero también de otros menos conocidos como acronym, address, blockquote, así como los atributos lang, cite, class, id, etc.

- Metadatos. Esto implica usar elementos y propiedades que cumplen funciones de metadatos: title de la sección head; el atributo title en todos los elementos que se puedan beneficiar del mismo, como los enlaces; el atributo alt en imágenes y por supuesto, los elementos meta con los atributos name y content en la sección head.
En realidad el uso adecuado de los elementos y atributos que forman parte de html permite llevar aún más lejos el marcado o la codificación semántica de las páginas (por ejemplo, no hemos mencionado las listas de definiciones, ni los atributos de la tablas, etc.), pero creemos que lo anterior es una buena indicación de todas estas posibilidades.

\section{"Estos tres productos corresponden a las tres formas principales de crear y publicar contenidos en internet: editores offline, editores online y servidores de blogs"}

\section{Tres formas de publicar en la web}

Los tres sistemas que presentamos aquí (NVU, Page y Blogger) corresponden a las tres formas principales en las que es posible crear y publicar contenidos en internet actualmente: editores offline, editores online y servidores de blogs.

Los editores offline como $N V U$ son el sistema más tradicional. Es el medio que proporciona mayor flexibilidad y posibilidades de control al autor, pero requiere una condición, disponer previamente de espacio en un servidor web, y un paso adicional aparentemente simple, como es el hecho de subir los documentos al servidor, pero que se comporta como una barrera real.

Los editores online como Pages son aplicaciones en línea, muy en la clave de la web 2.0, que unifican en una sola operación la creación y mantenimiento de las páginas y su publicación.

Por último, los servidores de blogs como Blogger son una variedad de la edición online. Al igual que en el segundo caso, los dos pa- sos comentados están unificados en una sola operación.

En los dos últimos sistemas la operación de actualizar y subir los archivos al servidor es transparente, lo que sin duda explica su popularidad. Pero también presentan mayores limitaciones comparados con el uso de un editor de páginas. Es cierto que éstos últimos después necesitan un cliente de ftp y subir los documentos por separado (aunque algunos programas, como el propio $N V U$ permiten realizar esta operación desde el mismo editor).

\section{1. $N V U$}

\subsubsection{Contexto}

$N V U$ (se pronuncia "n-view") es un programa multiplataforma, esto es, funciona en Windows, Mac y Linux, que se distribuye de forma gratuita bajo licencia open source. Está basado en Mozilla Composer, el editor de páginas web que incluía tradicionalmente la suite Mozilla, un tanto abandonada últimamente debido al éxito de Firefox.

Está disponible en diversos idiomas y puede descargarse una versión en castellano en el sitio del Proyecto Nave.

http://www.proyectonave.es/ productos $/ n v u /$

\subsubsection{Prestaciones}

Sus características principales son las siguientes:

- Es un editor de páginas de tipo wysiwyg, es decir, funciona en modo gráfico, lo que significa que con $N V U$ se pueden crear páginas web sin necesidad en teoría de conocer el lenguaje html. Esto en realidad nunca es cierto del todo, ni referido a $N V U$ ni a ningún otro programa de este tipo (Dreamweaver, por ejemplo).

- Es un editor también en código fuente, con dos tipos de vistas: la visión clásica y otra mediante iconos que facilita el trabajo con los elementos html de la página. 
- Incorpora un editor de hojas de estilo (css).

- Presenta un sencillo gestor de sitios que incluye a su vez un cliente ftp que facilita las operaciones básicas de las páginas en el servidor.

\section{"Lo mejor de NVU probablemente sea su editor de hojas de estilo y la posibilidad de editar las etiquetas html en un modo especial exclusivo mediante iconos"}

\subsubsection{Estándares}

Las mejores características de $N V U$ probablemente sean su editor de hojas de estilo y la posibilidad de editar las etiquetas html en un modo especial actualmente exclusivo de este programa mediante iconos que las representan (antes lo había usado un software ahora ya desparecido). Las figuras 2 y 3 ilustran estos dos aspectos del programa.

\subsubsection{Conclusiones}

En síntesis, es un completo sistema de creación de páginas y sitios web en el que destacan, además de las cuestiones señaladas, y siempre desde el punto de vista que hemos presentado al inicio, las facilidades que proporciona para utilizar estándares web. En concreto, es posible definir el programa de manera que cree los documentos en formato Xhtml Strict o Transitional (además de html 4.01), a diferencia de algunos programas comerciales que ni siquiera prevén la generación de una declaración doctype. Otra de sus buenas bazas es su preocupación por aspectos de accesibilidad, ya que no permite incorporar elementos gráficos sin añadir metadatos a los mismos.

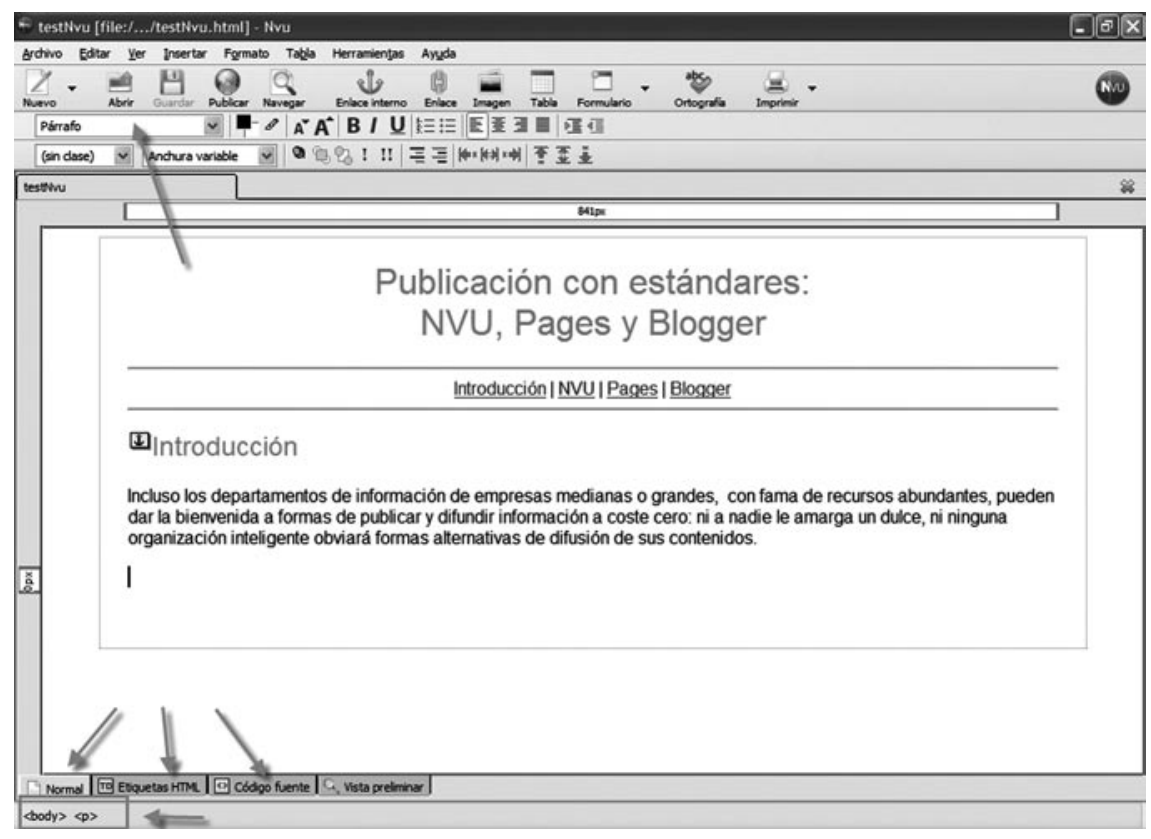

Figura 1. La ventana de edición en modo wysiwyg de NVU. Las flechas destacan (arriba) el desplegable para seleccionar y aplicar elementos al contenido así como (abajo) las pestañas que dan acceso a las diversas vistas y finalmente la barra de estado donde vemos el código $\mathrm{h} \mathrm{tml}$ que corresponde al lugar donde está situado el cursor.

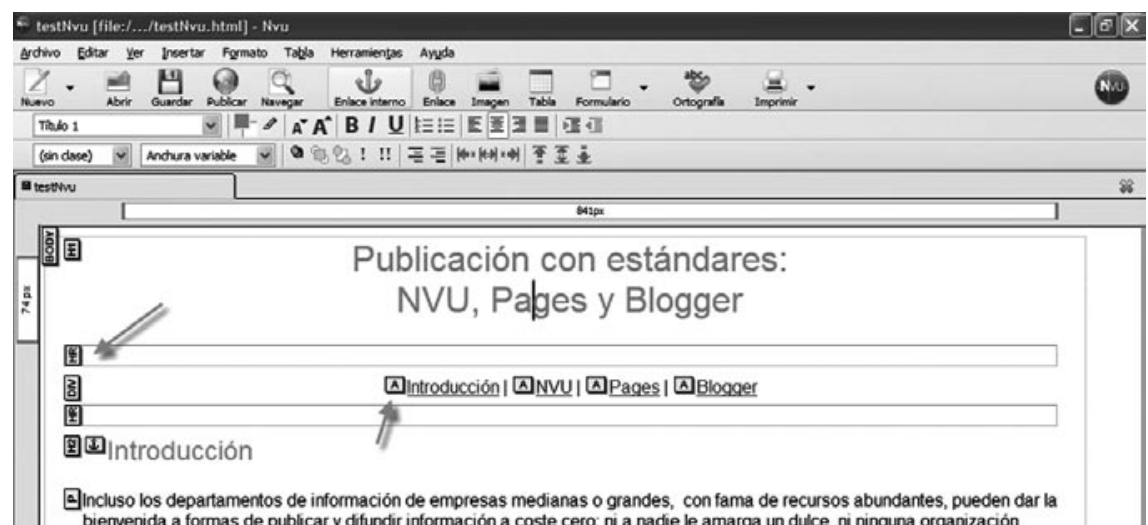

Figura 2. Editor de hojas de estilo de NVU. Mediante un sistema de pestañas y ventanas de selección se define cualquier aspecto de estilo de los elementos html de la página, así como estilos de clase (class) y de identidad (id) con bastante comodidad.

Uno de los principales aspectos del programa es la presentación del código fuente. $N V U$ es capaz de generarlo limpio, sin elementos depreciados. También es posible aplicar estilos css en lugar de propiedades de forma automática, pero el resultado aparece presentado de una forma completamente desordenada, sin posibilidad de aplicarle buenas prácticas no ya a nivel de indentaciones (sangrías), sino simplemente de separación de bloques de líneas, etc. Con todo, cabe admitir que es una cuestión bastante menor, ya que disponemos de la vista de iconos ya mencionada.
El mayor problema de este producto es que no está clara su continuidad. Las grandes ilusiones que generó la aparición a mediados de 2005 de un editor de páginas web que podía medirse con Dreamweaver, se van enfriando a medida que pasa el tiempo y no solamente no aparecen nuevas actualizaciones sino que en su web oficial ni siquiera se anuncian planes futuros. $\mathrm{Al}$ parecer, pero no existe ninguna declaración oficial sobre ello, el responsable principal de $N V U$ está trabajando para un nuevo desarrollo que recuperará el nombre original de Composer y que se ofrecerá 


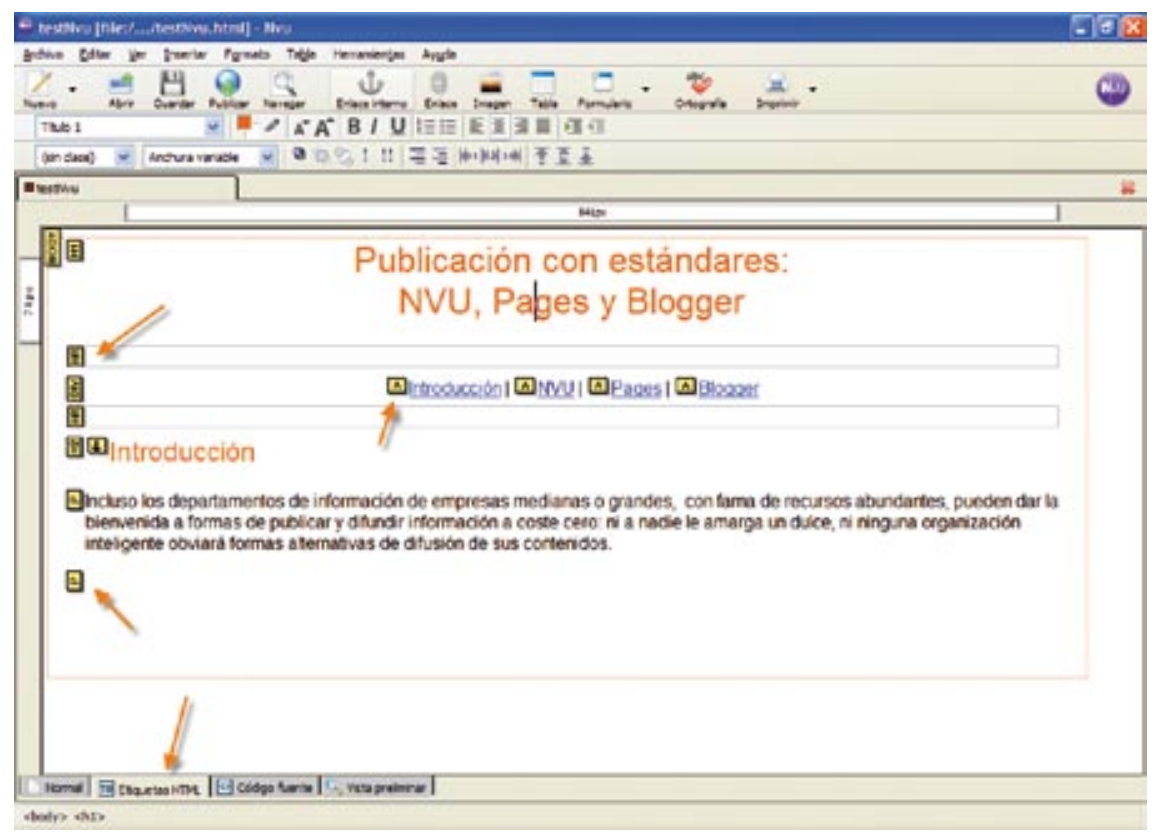

Figura 3. Las flechas señalan algunos de los iconos. Cada uno de ellos es un elemento html (a, $\mathrm{p}$, hr, etc.). La disposición de los iconos muestra también la estructura jerárquica de la página. Haciendo clic sobre ellos se abre una ventana que permite añadir atributos a los elementos.

como parte una futura versión de la suite Mozilla que se denominará SeaMonkey. Mientras tanto, ante la aparente parálisis del proyecto, ha aparecido una versión no oficial denominada Kompozer que soluciona algunos errores del programa. Por tanto, se puede considerar que la última versión de $N V U$, fechada en julio de 2006, se denomina Kompozer.

\subsection{Pages}

\subsubsection{Contexto}

Es una aplicación online de Google que une un sistema de edición de páginas con uno de publicación online con un espacio de alojamiento (100 MB), y todo ello de forma gratuita.

\section{http://pages.google.com}

\subsubsection{Prestaciones}

Es uno de los sistemas de publicación web más rápidos del mercado y si lo comparamos con soluciones similares de pago, aunque posiblemente no sea la mejor ni la más completa, sí puede decirse que es una de las mejores, ofreciendo una calidad y un nivel de prestaciones que hubiera sido inimaginable encontrar, incluso bajo algún coste,

\subsubsection{Estándares}

Pages proporciona una forma tal de cambiar la apariencia de la página que de no existir la tecnología de las hojas de estilo parecería magia. Visto de otra forma, si alguien quisiera demostrar las ventajas de la separación entre contenido y forma no tendría más que acudir al formulario que permite cambiar tanto la apariencia (fuente, color, background, etc.) como la estructura (layout) con un simple clic. La razón no es otra que un uso magistral de hojas de estilo.

\section{"Pages proporciona una forma tal de cambiar la apariencia de la página que de no existir la técnica de las hojas de estilo parecería magia"} el formato cualquiercosa.googlepages.com, del espacio de hospedaje correspondiente (los $100 \mathrm{MB}$ ya mencionados) y de una primera página con un notable nivel de diseño, es cuestión de pocos minutos.

Las figuras 4 y 5 muestran el aspecto típico de un sitio creado de esta forma y la aplicación online que permite elaborar y modificar las páginas.

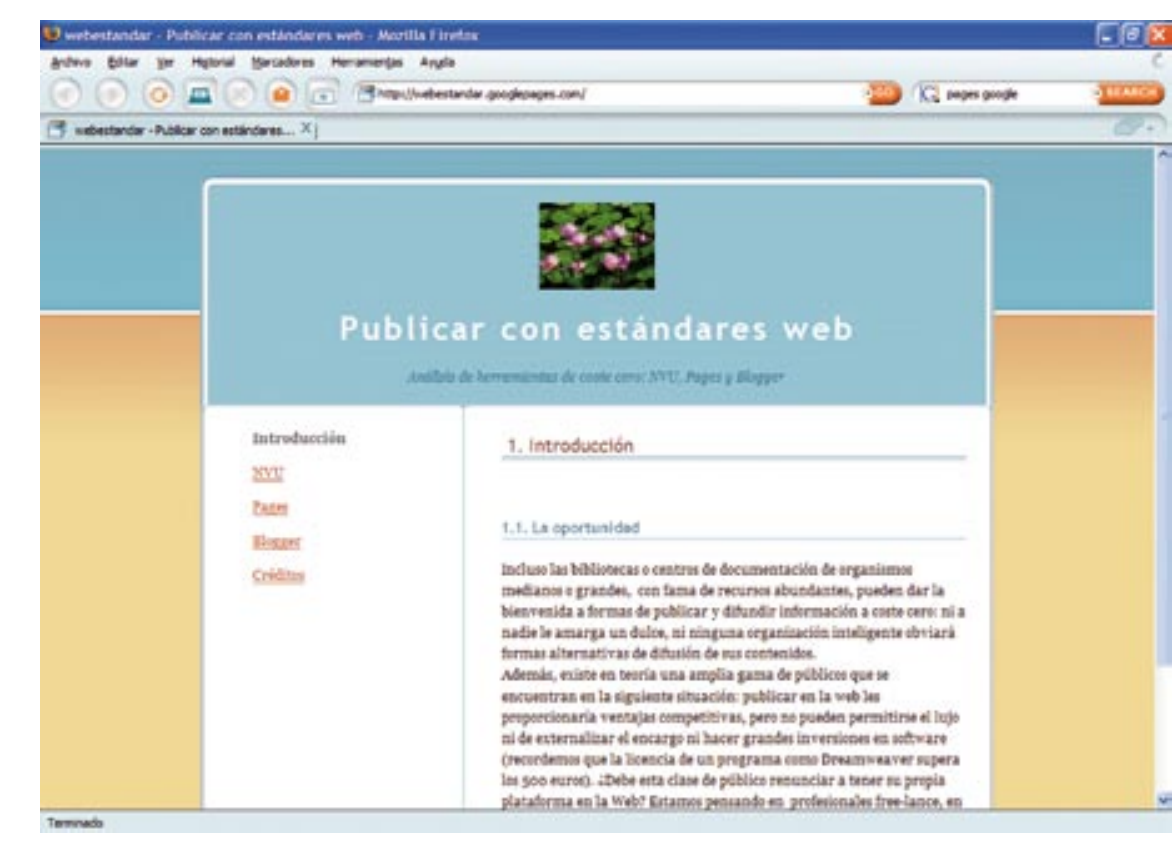

Figura 4. La página principal de un sitio creado con Google Pages como test para este artículo.

En concreto, si examinamos el código fuente de una página creada con Pages podemos ver que utiliza html 4.01 Transitional oportunamente declarado en la declaración doctype correspondiente y hojas de estilo incrustadas en la cabecera de la página (elemento style). 




Figura 5. El editor online de Google Pages. Obsérvese a la izquierda el panel con las diversas opciones de edición de la página. Hemos añadido un pequeño gráfico arriba para mostrar la versatilidad del programa.

En este sentido, la estructura de las páginas está definida por hoja de estilo (no utiliza tablas, por ejemplo). La presentación de los elementos (body, $p, h 1, h 2$, etc.) está determinada también de esta manera. Sin embargo, con la barra de herramientas de la izquierda, el usuario puede modificar el aspecto de algún componente, por ejemplo, algún aspecto de la fuente y en ese caso Pages aplica atributos depreciados como font.

\subsubsection{Conclusiones}

Es un sistema de edición en línea extremadamente eficaz y que, por motivos de eficiencia -casi podríamos decir de supervivenciaaplica, y de forma muy brillante, la separación de contenido y presentación. Sin embargo, cuando el usuario recurre a las opciones de la barra izquierda para añadir formato, no se ha adoptado la política de $N V U$ de usar el atributo style, sino que, o bien aplica elementos no semánti$\cos (i)$ o bien atributos depreciados (font).

Facilita la edición del código fuente, con lo que el usuario podría cambiar los elementos o atributos depreciados por declaraciones de estilo en línea (atributos style) uno a uno, pero no puede optar por la opción más lógica de modificar la hoja de estilo para declarar estilos de clase o de $i d$ según conviniera. Se comprende muy bien la política de Pages de no permitir modificaciones en head, porque invalidaría su modelo de publicación. Sin embargo, hubieran podido optar por el sistema de añadir estilos en línea como respuestas a las modificaciones que aplica el usuario vía menú de la izquierda, o permitir algún grado de gestión de la hoja de estilo a través de menús, de forma que no existiera el peligro de destruir dicha hoja. Puede que en futuras ediciones sean más conscientes de la necesidad de trabajar con estándares al cien por cien. Hay motivos para suponer que puede ser así, según veremos en el caso del tercer $\mathrm{y}$ último sistema que analizaremos aquí.

\subsection{Blogger}

\subsubsection{Contexto}

Es un servidor de blogs que fue adquirido por Google hace ya algunos años. Ya era muy usado en su momento, pero el hecho de pasar a ser parte de Google lo ha conver- tido probablemente en el sistema de creación y publicación de blogs más popular.

Recordemos que blog es la contracción del término "web log". Una traducción literal, pero muy aburrida, sería la de "anotación web" o "anotaciones en la web" y una propuesta de traducción más imaginativa sería la de "cuaderno de bitácora". Suelen presentar una estructura lineal con alternativas laterales, generalmente con enlaces a otras webs. Es decir, un blog consiste en una o más páginas web con sus diferentes entradas o posts colocadas linealmente una a continuación de otra, con las más recientes en primer lugar. La navegación es muy sencilla, ya que suele consistir en avanzar por la página principal a base de clics en la barra de scroll del navegador, o a través de la ordenación cronológica de las entradas. Algunos sistemas de publicación pueden añadir una segunda navegación mediante un listado también presentado cronológicamente de los títulos de las diferentes entradas.

\subsubsection{Prestaciones}

Su creación y publicación es sencilla por definición. Es decir, sencillez y blog son dos palabras que van unidas. No es extraño por tanto que podamos afirmar que, si crear un sitio con Pages era fácil, hacerlo con Blogger es literalmente un juego de niños. Bastan tres pasos para crear uno, con una url con el formato cualquiercosa.blogspot. com, y es cuestión de minutos o de segundos crear, editar y publicar nuevas entradas en el blog.

\section{"Si crear un sitio con Pages era fácil, hacerlo con Blogger es literalmente un juego de niños"}




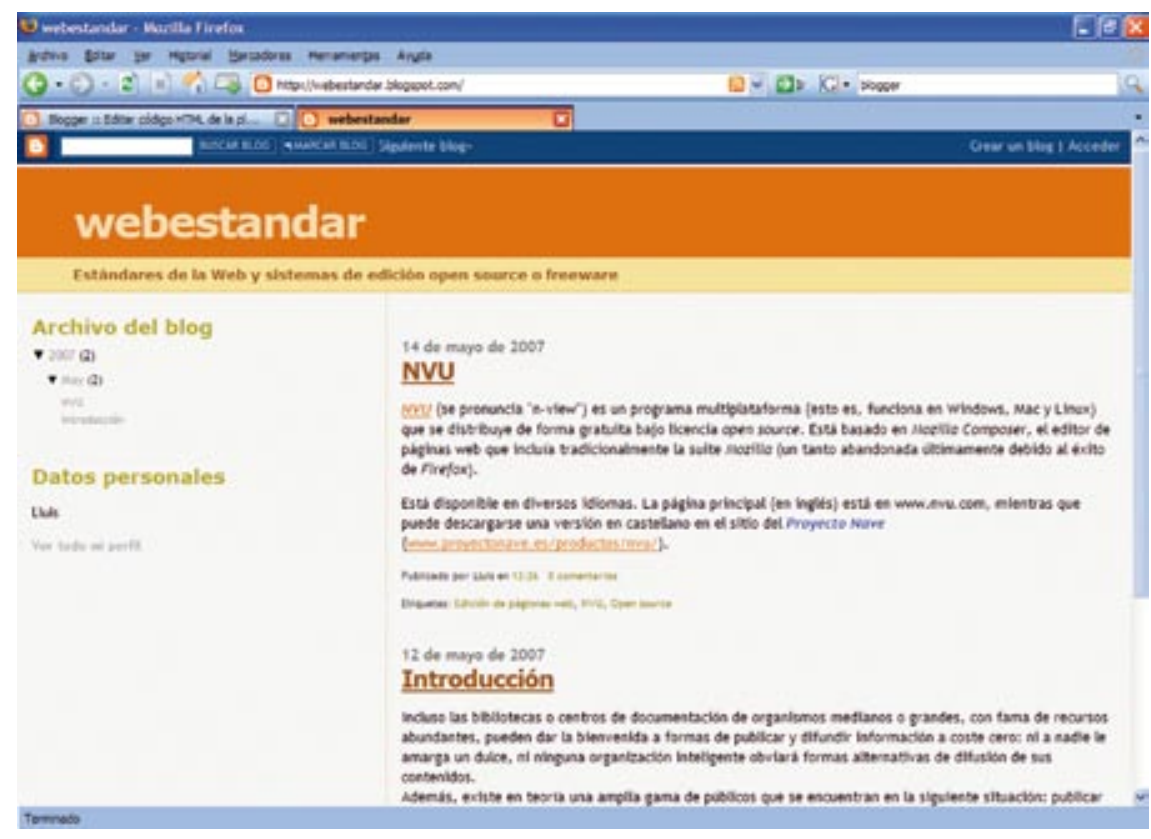

Figura 6. Aspecto típico de un blog creado con Blogger. Puede observarse un simple sistema de navegación (ordenado cronológicamente) a la izquierda.

Las figuras 6 y 7 muestran, respectivamente, el aspecto de un blog (creado para este artículo) y el sistema de edición de Blogger de una de las entradas:

\subsubsection{Estándares}

Utiliza Xhtml 1.0 Strict, según muestra la declaración doctype del código fuente que genera automáticamente el sistema. Además usa exclusivamente hojas de estilo tanto para la presentación de los distintos elementos de la página como para su estructura (layout).

A diferencia de Pages, las modificaciones puntuales que introduce el autor de la página utilizando las opciones disponibles en el sistema de edición en línea se traducen en estrictas declaraciones de estilo usando el atributo style. Al parecer, por tanto, el sistema supera en respeto a las estándares y las buenas prácticas a su primo hermano $\mathrm{Pa}$ ges.

Sorprendentemente, permite la edición directa y aparentemente sin limitaciones de la totalidad del código fuente, incluyendo por tanto la cabecera y por consiguiente la hoja de estilo que está incrustada en la propia página.

\subsubsection{Conclusiones}

Las pruebas realizadas muestran que Blogger genera un código totalmente exento de elementos depreciados así como su adhesión al nivel más exigente de cumplimiento de estándares Xhtml (la variación Strict). Es por esto que creemos que tal vez en el futuro Pages sea capaz de interpretar las modificaciones puntuales de los usuarios en las pá- ginas como declaraciones de estilo en línea en lugar de usar elementos depreciados.

Un paso adelante a favor de las buenas prácticas sería que en el futuro la hoja de estilo estuviera declarada en un archivo aparte y quedara vinculada mediante un elemento link. Sin duda, esto requeriría algo más de ingeniería si se deseara que esta forma de trabajar fuera transparente al usuario.

\section{Conclusiones generales}

Hemos visto tres medios para editar, y en su caso, publicar información en la Web. El primero es un editor de páginas $(N V U)$, lo cual requiere obtener de manera independiente los medios para publicar la información, mientras que los otros dos medios (Pages, Blogger) unen en un solo sistema la creación y la publicación así como aportan el servidor y el espacio correspondiente en el mismo. Esto último es indudable que constituye una ventaja en algunas situaciones, pero no en todas. En muchos casos se preferirá una aplicación como $N V U$ que no implica el uso de un determinado servidor con las limitaciones que conlleva.



Figura 7. Sistema de creación y modificación de entradas de Blogger. Una barra de botones permite la modificación de elementos de la entrada. 
Lo mejor es que, aunque son distintos, no son autoexcluyentes. Y lo más significativo es que en los tres casos existe la posibilidad de crear contenidos para la Web respetando estándares y observando algunas buenas prácticas elementales.

\section{"Lo más significativo es que en los tres casos se crean contenidos para la Web respetando estándares"}

Todos aquellos que crean que su actividad profesional puede benefi- ciarse de disponer de presencia en la Web o del hecho de dar a conocer informaciones útiles, no deberían frenar sus planes solamente por motivos económicos. Menos aún por razones prácticas o de oportunidad. El único freno que debería actuar es el de la falta de calidad de la información.

Así que, demos la vuelta a esta idea y veámoslo así: toda aquella persona u organización que esté en condiciones de difundir información útil para algún tipo de público, no debería dejar de hacerlo, al menos, insistimos, por motivos económicos o por la percepción (no siempre justificada como intentamos mostrar aquí) de falta de acceso a los recursos necesarios.

\section{Nota}

1. Recordemos que, por ejemplo, Adobe anuncia el precio de una licencia de Dreamweaver CS3 en algo más de $500 €$ (mayo 2007).

\section{Bibliografía}

Andrew, Rachel; Shafer, Dan. Html utopia: designing without tables using css. Collingwood: Sitepoint, 2006. ISBN 0-9752402-7-7.

Budd, Andy; Collison, Simon; Moll, Cameron. Css mastery: advanced web standards solutions. Berkeley: Friendsoft, 2007. ISBN 1-59059-614-5.

Griffiths, Patrick. The best-practice guide to xhtml and css. Berkeley: New Ridders, 2007. ISBN 0-321-31139-6.

Zeldaman, Jeffrey. Designing with web standards. Berkeley: New Riders, 2007. ISBN 0-32138555-1.

\section{Lluís Codina, Universidad Pompeu} Fabra.

lluis.codina@upf.edu

http://www.lluiscodina.com

\section{Vente al "nuevo" IweTel}

\section{Comité Asesor de IweTel}

La complejidad que ha adquirido nuestra profesión (sistemas de información, biblioteconomía, documentación, archivística, etc., y todas sus tecnologías informáticas y de telecomunicaciones) plantea muchas dudas a los administradores de la lista a la hora de decidir la aprobación de los mensajes.

Por ello hace unos meses se creó un Comité Asesor que define pautas de actuación, y en caso de duda aconseja sobre los contenidos que deben ser distribuidos:

$\begin{aligned} \text { Isidro F. Aguillo } & \text { (Cindoc) } \\ \text { Miguel Benito } & \text { (Borås, Suecia) } \\ \text { Mónica Caballo } & \text { (Jones Day) } \\ \text { Paco López Hernández } & (U C 3 M) \\ \text { Alejandro Martínez Andaluz } & (U P M) \\ \text { Reme Melero } & (\text { IATA }) \\ \text { Cristóbal Pasadas } & (\text { Univ. de Granada) } \\ \text { Honorio Penadés } & (U C 3 M) \\ \text { José R. Pérez Agüera } & (U C M) \\ \text { Francisco Tosete } & (U C 3 M) \\ \text { Cristóbal Urbano } & \text { (UB) } \\ \text { Salvador Zambrano } & \text { (Def. Pueblo Andaluz) }\end{aligned}$

Los administradores-moderadores de IweTel son Tomàs Baiget (Idescat) y Javier Leiva (Catorze.com e Informa)

El jefe del Servicio de Listas de RedIRIS es Jesús Sanz de las Heras (Red.es) 
En 2007 se ha creado el Centro Internacional para la Investigación en Estrategia y Prospectiva de la Información (CIEPI) con el objetivo de llevar a cabo estudios sobre la situación, tendencias y evolución de la información, sus técnicas, procesos, gestión, así como sobre el papel de sus profesionales.

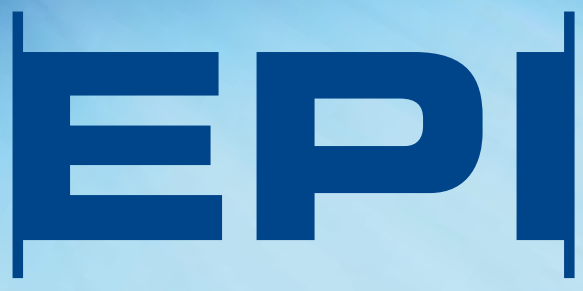

CIEPI está orientado a la investigación general, pero inicialmente sus trabajos se dirigirán más específicamente a la preservación de la información digital, la normalización, la evaluación y la visibilidad de la ciencia y de la e-ciencia, teniendo en cuenta especialmente sus aspectos sociales.

\section{CENTRO INTERNACIONAL PARA LA INVESTIGACIÓN EN ESTRATEGIA Y PROSPECTIVA DE LA INFORMACIÓN}

\section{A|ED| actuará principalmente जIP en dos sentidos:}

1. Fomento de la visibilidad de la producción científica.

2. Creación de un observatorio sobre tendencias de la información, para lo cual cooperará con el Grupo ThinkEPI.

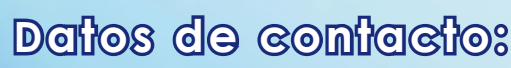

Fernanda Peset Universidad Politécnica de Valencia, Departamento de Comunicación Audiovisual,

Documentación e Historia del Arte, Camino de Vera, s/n 46022 Valencia

Tel.: 690682417

http://www.ciepi.org 